\title{
ERRATUM TO "INTEGRALITY AND ARITHMETICITY OF CO-COMPACT LATTICE CORRESPONDING TO CERTAIN COMPLEX TWO-BALL QUOTIENTS OF PICARD NUMBER ONE", ASIAN J. MATH., VOL. 8, NO. 1, 107-130, 2004*
}

\author{
SAI-KEE YEUNG ${ }^{\dagger}$
}

It has been called to the attention of the author that more details need to be provided in proving Subcase IICi (see (1) below). The purpose of this erratum is to provide such details, and correct some misstatements. The unexplained notations are the same as in the original paper.

(1) Page 117, line -4ff, add the following Lemma.

LEMMA 1. The set $f \circ \widetilde{p}\left(H_{a}\right)$ is convex.

Proof. Suppose $\Sigma$ is an apartment of the building such that $f \circ \widetilde{p}\left(H_{a}\right) \cap \Sigma$ is unbounded. We need to show that the image $f \circ \widetilde{p}\left(H_{a}\right) \cap \Sigma$ is isometric to $\mathbb{R} \cap \Sigma$ as a set with the Euclidean metric, if $\mathbb{R} \cap \Sigma \neq \emptyset$, where $H_{a}=\widehat{h^{-1}(a)}$. The earlier discussions in the article shows that it is true for each chamber in $\Sigma$. Without loss of generality, we may assume that the image of the ramification divisor $R$ by $f$ in an apartment $\Sigma$ containing an open set of $L_{a}$ is defined by $x_{2}-x_{3}=0$, so that $L_{a} \cap C$ for some chamber $C$ in $\Sigma$ is a line segment defined by $x_{2}-x_{3}=c_{a}$, a generic constant $\neq 0$. Hence $R$ is defined by $\omega_{2}-\omega_{3}=0$ on $M_{1}$. We are done if $\left.f\right|_{\widetilde{p}\left(H_{a}\right)}$ is non-singular, which implies that $f \circ \widetilde{p}\left(H_{a}\right)$ is isometric to $\mathbb{R}$. $\left.f \circ \widetilde{p}\right|_{H_{a}}$ has singularity only along another ramification divisor $R_{1}$ on $\widetilde{M}_{1}$, which is the stabilizer of an element $\iota \in \bar{W}$ of order 2 since its image lies in the wall of a building. Hence we may assume that the image of $R_{1}$ in $\Sigma$ is defined by $x_{1}-x_{2}=0$. As the local covering group generated by $\iota$ switches $d x_{1}$ and $d x_{2}$, we observe that $\left.f \circ \widetilde{p}\right|_{H_{a}}$ is extended beyond $\left.f \circ \widetilde{p}\right|_{H_{a}} \cap f \circ \widetilde{p}\left(R_{1}\right)$ in a unique way as a line segment in the adjacent chamber of $C$ in $\Sigma$ defined by $x_{1}-x_{3}=c_{a}^{\prime}$ for some constant $c_{a}^{\prime}$ determined by continuity.

Let $\tau$ be the global one form on $M_{1}$ annihilating $R_{1}$, defined locally by $1 / 2\left(\kappa_{1}+\right.$ $\left.\kappa_{2}\right)$, where $\kappa_{i}=\left(p^{*} f^{*}\left(d x_{i}\right) \otimes \mathbb{C}\right)^{1,0} . \tau$ is the pull back of a holomorphic one form $\tau_{o}$ on $E:=A / \operatorname{ker}(\tau)$ by $\alpha_{o}: M_{1} \stackrel{\alpha}{\rightarrow} A \stackrel{q}{\rightarrow} E$. Let $\eta=\alpha_{o}^{*} \Re\left(\tau_{o}\right)$. Fix $z_{o} \in H_{a}$ so that $\widetilde{p}\left(z_{o}\right)$ is a regular point of $f$. Define $\Phi: \widetilde{M}_{1} \rightarrow \mathbb{R}$ by $\Phi(z)=\int_{z_{0}}^{z} \eta$.

We claim that for each apartment $\Sigma$ for which $L_{a} \cap \Sigma$ is unbounded, there is a covering map $\Psi: \mathbb{R}=\Phi\left(\widetilde{M}_{1}\right) \rightarrow L_{a} \cap \Sigma$ which is a local isometry, so that $\Psi \circ \Phi(z)=$ $f \circ \widetilde{p}(z)$. Suppose $\Phi\left(z_{1}\right)=\Phi\left(z_{2}\right)$. Join $z_{i}$ to $z_{o}$ by a unique geodesic $\gamma_{i}$ on $H_{a}$ for $i=1,2$. It follows that for all $t$ on $\gamma_{1}$, there exists $w(t) \in \gamma_{2}$ varying continuously with respect to $t$ such that $\Phi(w(t))=\Phi(t) \in \mathbb{R}$. It suffices for us to show that $f \circ \widetilde{p}(t)=f \circ \widetilde{p}(w(t))$ for all $t \in \gamma_{1}$ by continuity argument. This is clearly so for $t$ in a small neighborhood of $z_{o}$ or a regular point of $f$ from definition of $\tau$. Hence we only need to make sure that the argument can be extended beyond the singularity set $\mathcal{S}$ of $f$. Observe that the spectral covering is defined equivariantly on $\widetilde{M}$ and it suffices for us to discuss on $M_{1}$. As formulated in $\S 2$ of the paper, $M_{1}$ is the desingularization of $M_{1 o}$, a connected component of $M_{1}^{\prime}$ defined by the single equation $\sum_{i=0}^{\ell} \alpha_{i}(x) t^{l-i}=0$ in $T^{*} M$, where $l=6$. Let $\pi: \widetilde{M}_{1} \rightarrow M_{1}$ be the universal covering.

\footnotetext{
*Received May 22, 2008; accepted for publication February 20, 2009.

${ }^{\dagger}$ Mathematics Department, Purdue University, West Lafayette, IN 47907, USA (yeung@ math.purdue.edu). The author was partially supported by a grant from the National Security Agency.
} 
Then $\alpha_{o}(\pi(t))=\alpha_{o}(\pi(w(t)))=Q \in \alpha_{o}(\mathcal{S})$. It follows that $t, w(t) \in \alpha^{-1}\left(E_{Q}\right)$, where $E_{Q}:=q^{-1}(Q)$ is connected in $A$. Here $E_{Q} \cap \alpha\left(R_{23}\right)=\emptyset$ from construction, where $R_{23}$ is defined by $\kappa_{2}-\kappa_{3}=0$ on $M_{1}$. The Albanese map $\alpha: M_{1} \rightarrow A$ descends to $\alpha_{o}: M_{1 o} \rightarrow A$, since from definition it contracts the fibers of the desingularization map $M_{1} \rightarrow M_{1 o}$. For the claim, it suffices for us to make the observation, to be proved below, that $\alpha_{o}^{-1}\left(E_{Q}\right)$ is irreducible and is a component of the ramification divisor containing both $\pi(t)$, and $\pi(w(t))$. Let $U_{t}$ and $U_{w(t)}$ be neighborhoods of $t$ and $w(t)$ on $H_{a}$. From the discussions in the first paragraph above, the extension of $\left.f \circ \widetilde{p}\right|_{U(t)}$ and $\left.f \circ \widetilde{p}\right|_{U(w(t))}$ through $f \circ \widetilde{p}(t)=f \circ \widetilde{p}(w(t))$ is uniquely determined by the order two stabilizer of $\alpha^{-1}\left(q^{-1}(Q)\right)$ in $M_{1}$. The claim follows from continuity.

To explain the observation, suppose there are two different components $D_{1 o}$ and $D_{2 o}$ of $\alpha_{o}^{-1}\left(E_{Q}\right)$, in which $D_{1 o}$ is a ramification divisor of $p$ and is defined by $\kappa_{1}-\kappa_{2}=$ 0 .

Sublemma. There cannot be any divisor on $M_{10}$ contracted by the Albanese $\alpha$.

To streamline our argument, we will postpone the proof of the sublemma to the end of this section. Since any negative curve on $M_{1}$ is contractible by Grauert's criterion (cf. [BHPV]), by considering universal properties of the Abanese map, $M_{1 o}$ does not support any divisor with negative self-intersection. Since the spectral covering $M_{1 o} \rightarrow M$ is (everywhere) finite, It follows that the image of a divisor in $M_{1 o}$ is finite as well. With no curves of negative self-intersection, by writing $\alpha^{*} E_{Q}=D_{1 o}+D_{2 o}+R^{\prime}$, where $R^{\prime}$ is the rest of the components in $\alpha^{*} E_{Q}$, it follows from $E_{Q} \cdot E_{Q}=0$ that actually $D_{1 o} \cdot D_{2 o}=0$ and hence $D_{1 o} \cap D_{2 o}=\emptyset$. On the other hand, their images as effective divisors on $M$ intersect at least at a point $P \in M$, which follows either from the fact that Picard number of $M$ is 1 , or that $D_{1 o}$ and $D_{2 o}$ are mapped to the same point on the quotient building $X / \rho(\Gamma)$. Hence there are at least two distinct points $X_{1}$ and $X_{2}$ at $p^{-1}(P) \cap\left(D_{1 o} \cup D_{2 o}\right)$. Since $\bar{W}=S_{3}$ acts on $M_{1}^{\prime}$, locally above a point $y \in p\left(D_{1 o}\right)$ on $M_{1}$, there are components of ramification divisors $\epsilon_{1}\left(D_{1 o}\right)=R_{23}$ and $\epsilon_{2}\left(D_{1 o}\right)=R_{13}$ given by $\kappa_{3}-\kappa_{2}=0$ and $\kappa_{1}-\kappa_{3}=0$, where $\epsilon_{i}=(i 3) \in S_{3}, i=1,2$. If $D_{1 o}$ meets neither $\epsilon_{1}\left(D_{1 o}\right)$ nor $\epsilon_{2}\left(D_{1 o}\right), \alpha^{-1}\left(E_{Q}\right)$ is not meeting any ramification divisor and hence is regular everywhere, which implies that image $f\left(p\left(\alpha^{-1}\left(E_{Q^{\prime}}\right)\right)\right)$ for a point $Q^{\prime}$ near $Q$ is a straight line in $\Sigma$ and we are done. Hence assume that $D_{1 o}$ meets, say $\epsilon_{1}\left(D_{1 o}\right)$ on $M_{1}^{\prime}$ at $X_{1}$. As $\omega_{1}=\omega_{2}=\omega_{3}$ at $X_{1}$ from definition, $\epsilon_{2}\left(D_{1 o}\right)$ passes through $X_{1}$ as well. It follows that for a generic point $X^{\prime}$ in a small neighborhood $U$ of $X$ in $M_{1}$, the cardinality of $U \cap p^{-1}\left(p\left(X^{\prime}\right)\right)$ is at least 6 and hence $U \cap p^{-1}\left(p\left(X^{\prime}\right)\right)=p^{-1}\left(p\left(X^{\prime}\right)\right)$. We conclude that there cannot be another divisor $D_{2 o}$ passing through another point in $X_{2} \in\left(p^{-1}(P)-\left\{X_{1}\right\}\right) \subset M_{1}$. This also follows from the fact that the orbit of $X_{1}$ by $S_{3}$ is just $X_{1}$ itself, implying that $\left\{X_{1}\right\}=p^{-1}(P)$ as a set.

Hence the claim is valid. From the claim, a connected unbounded component of $L_{a} \cap \Sigma$ is covered isometrically by $\mathbb{R}$. Recall also that $H_{a} \cap R=\emptyset$ from definition, since their image in $A$ are disjoint fibers of $\pi: A \rightarrow E$ in notation of the article. For a connected component $\widetilde{R}$ of the pull back of $R$ to $\widetilde{M}_{1}$, it follows that $\left(L_{a} \cap\right.$ $\left.\rho\left(p_{*}\left(\pi_{1}\left(M_{1}\right)\right)\right) f(\widetilde{R})\right) \cap \Sigma=\emptyset$, here $\rho\left(p_{*}\left(\pi_{1}\left(M_{1}\right)\right)\right) f(\widetilde{R}) \cap \Sigma$ forms an infinite number of equally spaced parallel straight lines given by $x_{2}=x_{3}+k$ for different integers $k$ when we represent $\Sigma$ as $x_{1}+x_{2}+x_{3}=0$ in $\mathbb{R}^{3}$. Hence the connected set $L_{a}$ lies in a strip confined by two such parallel lines as above. From the proof of the claim, $L_{a}$ can bend only at intersection with some lines of form $x_{1}=x_{2}+c$ or $x_{1}=x_{3}+d$ for some integers $c$ and $d$ in a unique way as described earlier. Recall that the stabilizer of $G$ acts on $\Sigma$ by the affine Weyl group $W:=\mathbb{Z}^{2} \rtimes S_{3}$. Bending along $x_{1}=x_{2}+c$ or $x_{1}=x_{3}+d$ 
correspond to reflections (12), (23) $\in S_{3}$ respectively. The fact that no bending occurs along any line $x_{2}=x_{3}+e$ for some integer $e$ means that $\rho\left(\pi_{1}\left(h^{-1}(a)\right)\right) \cap W$ does not contain element of type $(a,(13)) \in \mathbb{Z}^{2} \rtimes S_{3}$. Hence only one of (12) of (23) may occur as element $h$ for some element $(b, h) \in\left(\mathbb{Z}^{2} \rtimes S_{3}\right) \cap \rho\left(\pi_{1}\left(h^{-1}(a)\right)\right)$, for otherwise their product gives rise to some element $\left(b^{\prime},(13)\right) \in\left(\mathbb{Z}^{2} \rtimes S_{3}\right) \cap \rho\left(\pi_{1}\left(h^{-1}(a)\right)\right)$. Suppose $(b,(12))$ does appear as an element in $\left(\mathbb{Z}^{2} \rtimes S_{3}\right) \cap \rho\left(\pi_{1}\left(h^{-1}(a)\right)\right)$. Then for all elements $(b, h) \in\left(\mathbb{Z}^{2} \rtimes S_{3}\right) \cap \rho\left(\pi_{1}\left(h^{-1}(a)\right)\right), h$ can only take the value of $(1)$ or $(12)$. We now show that the latter cannot happen. Suppose on the contrary that $\left(b_{1},(12)\right)$ belongs to $\left(\mathbb{Z}^{2} \rtimes S_{3}\right) \cap \rho\left(\pi_{1}\left(h^{-1}(a)\right)\right)$ for some $b_{1} \in \mathbb{Z}_{2}$. It implies that a bending of $l_{a}$ does occur at some $x_{1}=x_{2}+c$. By taking composition with another element in the infinite group $\left(\mathbb{Z}^{2} \rtimes S_{3}\right) \cap \rho\left(\pi_{1}\left(h^{-1}(a)\right)\right)$ if necessary, we may assume that $b_{1}$ is non-trivial. Taking powers of such elements shows that $L_{a}$, which is connected and unbounded in $\Sigma$, cannot be confined to a strip between $x_{2}=x_{3}+k_{1}$ and $x_{2}=x_{3}+k_{2}$ for some fixed integers $k_{1}$ and $k_{2}$, by considering a sequence of line segments in the apartment which can only be parallel either to $x_{2}=x_{3}$ or $x_{1}=x_{2}$ and bend only at intersection with lines of form $x_{1}=x_{2}+c_{i}, c_{i} \in \mathbb{Z}$ in the way determined by the claim. Hence $h=1$ and $\rho\left(\pi_{1}\left(h^{-1}(a)\right)\right) \cap W$ can only act by translation, corresponding to the first factor in $\mathbb{Z}^{2} \rtimes S_{3}$. Since $L_{a}$ is isometric to $\mathbb{R}$ from the claim, it means that $L_{a}$ sits as a straight line in $\Sigma$. This concludes the proof of the Lemma.

Proof of Sublemma. Consider the Stein factorization of the Albanese map $\alpha: M_{1 o} \stackrel{a}{\rightarrow} N \stackrel{b}{\rightarrow} A\left(M_{1 o}\right)$, where $a$ has connected fibers and $b$ is finite. We claim that $N$ has only rational singularities, following an observation of Jungkai Chen in private communication. Assume on the contrary so that $R^{1} a_{*} \mathcal{O}_{M_{1}} \neq 0$. From our setting, $R^{1} \alpha_{*} \mathcal{O}_{M_{1 o}}$ is supported at isolated points on $A\left(M_{1 o}\right)$. It follows that $h^{0}\left(A\left(M_{1 o}\right), R^{1} \alpha_{*} \mathcal{O}_{M_{1 o}} \otimes Q\right) \neq 0$ for all $Q \in \operatorname{Pic}^{o}\left(A\left(M_{1 o}\right)\right)$. From Leray spectral sequence and projection formula, it follows that $h^{1}\left(M_{1 o}, \mathcal{O}_{M_{1 o}} \otimes a^{*} Q\right) \neq 0$. From Serre Duality, we conclude that for all $P \in \operatorname{Pic}^{o}\left(M_{1 o}\right), h^{1}\left(M_{1 o}, \omega_{M_{1 o}} \otimes P\right) \neq 0$. This however contradicts the generic vanishing theorem $(\mathrm{cf} .[\mathrm{CH}])$, which implies that the codimension of $V^{1}:=\left\{P \in \operatorname{Pic}^{o}\left(M_{1 o}\right) \mid h^{1}\left(M_{1 o}, \omega_{M_{1 o}} \otimes P\right) \neq 0\right\}$ in $\mathrm{Pic}^{o}$ is at least 1 and concludes the proof of the claim. It follows from the claim and Artin's Criterion (cf. [BHPV], Theorem (3.2)) that any divisor contracted by $\alpha$ and hence $a$ has to be a rational curve, which descends to $M$ by the finite map $M_{1 o} \rightarrow M$. However $M$ is hyperbolic and hence does not contain any rational curve. Sublemma follows.

(2) The statement on page 118, line -5ff: 'However... non-empty' is not correct. Subcase IICii can be handled by the following argument. Let $D_{o}$ be codimension one component in $R_{p}$ contracted by $\alpha$. As $\alpha\left(M_{1}\right)$ is smooth, $D_{o}$ is a rational curve, which implies that $p\left(D_{o}\right)$ is a point on $M$ since $M$ is hyperbolic. Hence every codimension one component in the $R_{p}$ is contracted by $p$. Recall that $M^{\prime}$ is defined in $\S 2$, page 112 , by an equation of the form $\sum_{i=0}^{m} \alpha_{i} t^{m-i}=0$, on which the ramification divisor of $\left.p\right|_{M_{1}^{\prime}}$ is obtained by identifying two roots of the equation. Its image in $M$ is the zero set of the single polynomial given by the discriminant of the defining polynomial and hence is of codimension one. As $\left.p\right|_{M_{1}^{\prime}}: M_{1}^{\prime} \rightarrow M$ in $\S 2$ is unramified in dimension one, we conclude that the spectral covering is an unramified covering of $M$.

The followings are some minor corrections.

(3) Page 120 , line -9ff, replace ' $q^{-1}(p) \cap B=\emptyset$ ' by ' $q^{-1}(p) \cap B$ is a finite number of points.'

(4) On page 123 , line -1ff and 124 , line $9 \mathrm{ff}$, after ' $K_{\widetilde{M}_{\sigma}}=3 H_{M_{\sigma}}$ ', add 'modulo torsion' 
(cf. Lemma 8.3 of $[\mathrm{K}]$ ).

(5) On page 124, line 18ff, after '... continuous.', add 'Note that according to Lemma 6.1.6 and 6.1.7 and their proofs in [Z], we conclude from integrality of $\rho(\Pi)$ that $G$ can be defined over a real number field $k$.'

The author would like to express his gratitude to the referee for painstaking readings and helpful comments.

\section{REFERENCES}

[BHPV] W.P. Barth, K. Hulek, A.M. Chris, And A. VAn de Ven, Compact complex surfaces, Second edition. Ergebnisse der Mathematik und ihrer Grenzgebiete, 3, Folge. A Series of Modern Surveys in Mathematics 4, Springer-Verlag, Berlin, 2004.

[CH] J.A. Chen And C.D. HACON, Pluricanonical maps of varieties of maximal Albanese dimension, Math. Ann., 320:2 (2001), pp. 367-380.

[K] J. Kollár, Shafarevich maps and automorphic forms, Princeton University Press, Princeton (1995).

[Z] R. Zimmer, Ergodic Theory and Semisimple groups, Birkhäuser, 1984. 